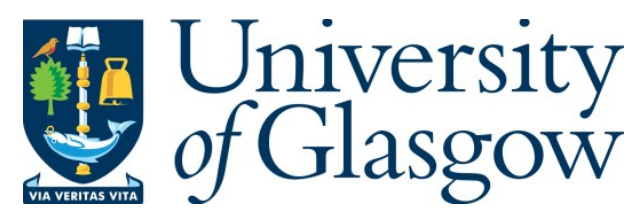

Sambo, Y. A., Heliot, F., and Imran, M. A. (2015) A survey and tutorial of electromagnetic radiation and reduction in mobile communication systems. IEEE Communications Surveys and Tutorials, 17(2), pp. 790-802.

There may be differences between this version and the published version. You are advised to consult the publisher's version if you wish to cite from it.

http://eprints.gla.ac.uk/136074/

Deposited on: 1 February 2017

Enlighten - Research publications by members of the University of Glasgow http://eprints.gla.ac.uk 


\title{
A Survey and Tutorial of Electromagnetic Radiation and Reduction in Mobile Communication Systems
}

\author{
Yusuf A. Sambo, Student Member, IEEE, Fabien Héliot, Member, IEEE, \\ and Muhammad Ali Imran, Senior Member, IEEE
}

\begin{abstract}
This article provides a survey and tutorial of electromagnetic (EM) radiation exposure and reduction in mobile communication systems. EM radiation exposure has received a fair share of interest in literature; however, this work is one of the first to compile the most interesting results and ideas related to EM exposure in mobile communication systems and present possible ways of reducing it. We provide a comprehensive survey of existing literature and also offer a tutorial on the dosimetry, metrics, international projects as well as guidelines and limits on the exposure from EM radiation in mobile communication systems. Based on this survey and given that EM radiation exposure is closely linked with specific absorption rate (SAR) and transmit power usage, we propose possible techniques for reducing EM radiation exposure in mobile communication systems by exploring known concepts related to SAR and transmit power reduction of mobile systems. Thus, this paper serves as an introductory guide for EM radiation exposure in mobile communication systems and provides insights towards the design of future low EM exposure mobile communication networks.
\end{abstract}

Index Terms-EM radiation exposure, mobile communication systems, specific absorption rate, power density, future networks.

\section{INTRODUCTION}

There has been a remarkable growth in mobile communications since its introduction in the 1970s. The evolution of mobile phones from mere voice communication devices to ubiquitous data-hungry smartphones that partake in our daily lives has resulted in continuous network upgrades and large-scale deployment of base stations (BSs) to support the increasing demand for bandwidth and provide coverage to mobile subscribers. The total number of mobile subscribers is projected to be over 8 billion by 2016 [1], which means that more BSs would have to be deployed to support the evergrowing increase in mobile users. However, given that mobile communication systems rely on radiofrequency (RF) waves to operate, the ubiquitousness of mobile communication systems raises the level of electromagnetic (EM) radiation exposure to the public. Thus, concerns about possible adverse health effects due to exposure to RF radiation from mobile communication systems are also growing [2], [3]. These worries are borne out of the popularity of mobile phones and the increased deployment of BSs closer to the general public in order to support the growing demand for bandwidth.

Y. A. Sambo, F. Héliot and M. A. Imran are with the Institute for Communication Systems (ICS), home of the 5G Innovation Centre, University of Surrey, Guildford, GU2 7XH, United Kingdom. (Email: \{yusuf.sambo, f.heliot, m.imran\}@ surrey.ac.uk).

\section{A. Basis for EM Radiation Exposure Guidelines and Limits}

There have been many public debates about the siting of BSs as well as the short and long-term implications of exposure to EM emissions from these BSs on humans and animals [4]. The most reoccurring questions focus on the EM radiation exposure levels within the vicinity of the BSs, effects of increased and indiscriminate deployment of BSs and EM regulatory compliance of BS antennas. Although several reports suggest that EM radiation from BSs are too low to produce any health risks (as long as there is no direct contact with the antennas), there are still concerns about the long-term effects of such exposure. Ironically, most of the concerns about EM exposure have been from the BS, even though emissions from mobile phones present a greater health risk since the antennas are closer to the human body, usually the head, when in operation. A 2011 report by the international agency for research on cancer (IARC) of the world health organization (WHO) concluded that EM radiation is possibly carcinogenic to humans and classified it as Group 2B, a category used when there is limited evidence of carcinogenicity in humans [5]. As a result of this, several international and national EM radiation exposure guidelines and limits have been introduced and adopted by relevant regulatory bodies.

\section{B. International Projects/Consortiums on EM Radiation Ex- posure}

In this subsection, some international projects with the objectives of investigating possible health effects, reducing EM radiation exposure and/or reducing the transmit power of mobile communication systems are presented.

\section{- The International EMF Project}

The international EMF project is an ongoing project established by the WHO in 1996 to assess possible health effects of EM radiation exposure. The project consists of WHO member states, departments of health and representatives of national institutions concerned with overseeing EM radiation exposure. Some key objectives of the project are to aid the development of internationally acceptable standards on EM radiation exposure, provide an international response to concerns about possible health effects of EM radiation exposure and to make a status report on EM radiation exposure based on scientific literature. Also, the project aims to encourage a dedicated research programme on EM radiation exposure in partnership with funding agencies and incorporate the research results into the environmental health criteria monographs 
of the WHO, and advise the international community on any hazards due to EM radiation exposure [6].

\section{- Low EMF Exposure Future Networks (LEXNET)}

The low EMF exposure future networks (LEXNET) project is a consortium of 17 partners from 9 European countries, consisting of industrial partners, network operators, research centres and universities among others. The LEXNET project is the first and only project with the aim of reducing EM radiation exposure of future communication systems. It is supported by the European Commission $7^{\text {th }}$ framework programme (FP7), under the information and communication technologies (ICT) work programme. The project has the target of reducing public exposure to EM radiation by at least $50 \%$ without compromising the quality of service (QoS) of the networks nor the quality of experience (QoE) perceived by the users. Other tasks of the LEXNET project are to define a novel and more realistic index for assessing human exposure to EM radiation and to build numerical and experimental tools to assess this index. In addition, the project will identify and propose cross layer techniques capable of reducing EM radiation exposure of future wireless networks. The project started in November 2012 and will last until October 2015 [7].

\section{- Energy Aware Radio and neTwork tecHnologies} (EARTH)

The EARTH project was a European Commission FP7 project that had the target of reducing the energy consumption of mobile systems by at least 50\% [8]. It was a consortium of 15 industrial and academic partners from 10 European countries. The project was concluded in June 2012 after a duration of 30 months. Some results of the EARTH project include the development of new network management mechanisms, energy efficient network architectures and deployment strategies, and new radio and network resource management protocols for multi-cell cooperative networks. The EARTH project overachieved its target by designing integrated solutions that provide energy savings of up to $70 \%$. Although the targets of the EARTH project did not include EM radiation exposure reduction, some of their proposed works provide a reduction in the transmit power of mobile systems which could result in lower levels of EM radiation exposure [9] and [10].

\section{- GreenTouch}

GreenTouch is an international consortium of experts from the industry and the academia dedicated to improving the energy efficiency of wireless communication networks by a factor of 1000, compared to 2010 levels. It has a target of specifying the architecture and showing fundamental components needed to considerably improve network energy efficiency by 2015. However, like the EARTH project, the GreenTouch project also directly focuses on energy efficiency but the proposed solutions could have the potential of reducing EM radiation exposure levels [11].
Although the exposure to EM radiation has been well investigated, there is no consensus on the health effects of EM radiation exposure from mobile communication systems. It is, therefore, imperative to provide a comprehensive review of existing literature, dosimetry, metrics, international projects as well as guidelines and limits on the exposure from EM radiation in mobile communication systems. Whereas the EM radiation exposure from mobile communication networks and devices are generally below regulatory limits [12], the precautionary approach is to substantially minimize the potential health hazards due to EM radiation exposure. Hence, the aim of this paper is not only to provide a clear-cut survey of the existing research works on EM radiation, but to also identify possible techniques for the design of EM radiation exposureaware future networks. These techniques have been proven to reduce the SAR and/or transmit power of mobile communication systems, and consequently, EM radiation exposure levels due to the direct relationship between transmit power, SAR and EM radiation exposure.

The contributions and organisation of this paper are presented as follows:

- In section II, we describe the different EM field regions around a transmitting antenna based on distance and how they affect EM radiation exposure calculations. We then present the usual metrics for evaluating the EM radiation exposure in the various EM field regions and how this exposure is assessed for different body parts.

- We describe the EM radiation exposure in the mobile communication system in section III. We begin with a brief description of the radiofrequency spectrum and the mobile communication system. We then explain how different network topologies, duration of exposure and location of the mobile user affect EM radiation exposure.

- We present, in section IV, the dosimetry of EM radiation exposure based on the method of evaluation. We also discuss the adopted EM radiation exposure guidelines and limits for mobile communication systems. We then examine EM radiation exclusion zones based on the prescribed limits to reduce EM radiation exposure, as a precautionary approach.

- In section $\mathrm{V}$, we identify and discuss some possible EM radiation exposure reduction techniques in mobile communications. The techniques are presented based on their ability to reduce SAR and/or transmit power of mobile communication systems while keeping an acceptable QoS, as EM radiation exposure is directly related to transmit power in both uplink and downlink scenarios.

- Finally, section VI provides a summary and conclusion of this survey.

\section{EM RADIATION METRICS}

The transmit power and directivity of an antenna are the two main factors determining the EM radiation from the antenna in a certain direction. Omnidirectional antennas radiate power uniformly in all directions, while directional antennas radiate most of the energy in a given direction, depending on the gain of the antenna. The higher the gain of an antenna, the 


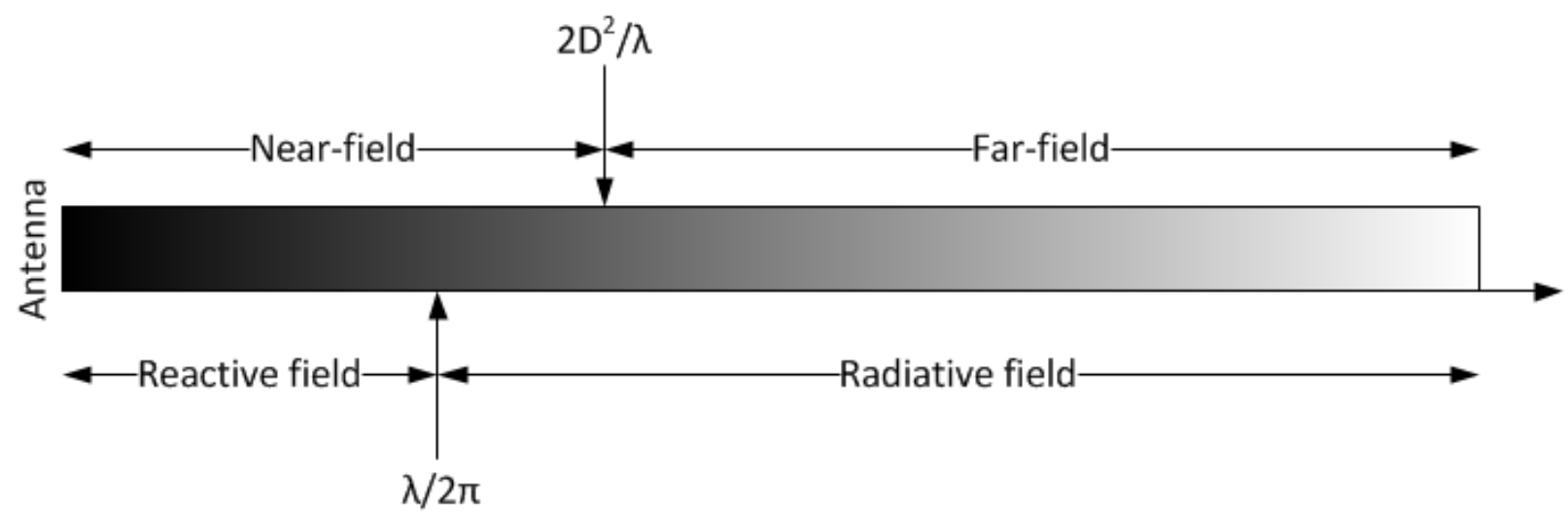

Figure 1. EM radiation fields

stronger the signal in the desired direction, when compared to an omnidirectional antenna.

All transmitting antennas have two regions that are defined by the electric and magnetic fields based on distance from the antenna, namely: the near-field and the far-field. Given that the near and far-fields behave differently in terms of their radiation pattern and decay, the EM radiation in these fields are evaluated by using two different metrics; the Specific Absorption Rate (SAR) is usually considered in the near-field, whereas in the far-field, power density is used.

Since BSs transmit moderate to high power over long distances and the mobile terminal transmits low power but close to the user, near and far-fields EM radiation will mainly impact the uplink and downlink scenarios, respectively, of mobile communication systems. Indeed, SAR and power density metrics are usually considered in the literature (e.g., [13] and [14]) for characterizing the uplink and downlink EM radiation exposure.

\section{A. Near-Field and Specific Absorption Rate (SAR)}

The near-field region refers to areas closer to the antenna. The near-field is also divided into two areas; the reactive and radiative areas, as shown in Fig. 1. The reactive nearfield region begins from the antenna up to the distance $\lambda / 2 \pi$, where $\lambda$ is the wavelength. Measurements in the reactive area are complicated to undertake given that the electric and magnetic fields are difficult to characterize in this area such that independent evaluation of these two quantities are usually performed. The energy dissipated within the reactive near-field region could easily be absorbed by the human body, thus, it serves as the major factor of exposure in the near-field [15]. The radiative area denotes the beginning of the far-field and the fields begin to radiate here. The strength of the fields in this region varies inversely with the cube of the distance from the antenna i.e., $1 /(\text { distance })^{3}$.

The SAR, in Watts per kilogramme of body weight $(\mathrm{W} / \mathrm{kg})$, is typically used to measure the EM radiation exposure level in the near-field region averaged over time, $t$ (minutes). SAR is a measure of the rate absorption of energy by the body when exposed to EM radiation. The EM exposure from mobile phones is mostly in the near-field as the phone is usually close to the body (and head in particular), therefore, SAR is used to evaluate such exposure over a certain period of time, $t$. The instantaneous EM radiation exposure in the near-field is given as $\mathrm{SAR} \times t$, expressed in $\mathrm{J} / \mathrm{kg}$. SAR is mathematically expressed as:

$$
\mathrm{SAR}=\frac{\delta}{\delta t}\left(\frac{\delta W}{\delta m}\right)=\frac{\delta}{\delta t}\left(\frac{\delta W}{\rho \delta V}\right)
$$

where $W, m, V$ and $\rho$ represent the energy absorbed by the body, mass, volume and density of the body, respectively [16].

SAR is measured differently for different body parts due to their rate of absorption. Therefore, the SAR metric is further subdivided into

1) Whole-body averaged $S A R$ (wbaSAR): This gives the ratio of the total power absorbed in the body and the mass of the body. It has been designated as the reference for EM radiation measurements [17]. For regulatory compliance, the wbaSAR is averaged over a period of 6 minutes. The wbaSAR can be expressed as:

$$
\operatorname{wbaSAR}=\frac{1}{M} \int_{R} \operatorname{SAR} \delta m=\frac{1}{M} \int_{R} \sigma|E|^{2} \delta V
$$

where $M, R$ and $V$ denote the total mass of the body, the region of the body and the total tissue volume of the body model [16].

2) Organ-specific averaged SAR (osaSAR): This is defined as the mass average of the SAR in a specfic organ or tissue in the body. The osaSAR portrays how EM fields are absorbed by specific parts of the body. The osaSAR is given as:

$$
\begin{aligned}
\text { osaSAR } & =\frac{1}{M_{\text {organ }}} \int_{\text {organ }} \operatorname{SAR} \delta m \\
& =\frac{1}{M_{\text {organ }}} \int_{\text {organ }} \sigma|E|^{2} \delta V
\end{aligned}
$$

where $M_{\text {organ }}$ denotes the mass of the organ or tissue under consideration [16].

3) Peak-spatial averaged SAR (psaSAR): This is defined as the maximum local SAR averaged over a specific mass of tissue, usually $10 \mathrm{~g}$, over a period of 6 minutes [16].

The SAR of all mobile devices is measured for regulatory compliance prior to device authorization or use [18]. SAR measurement involves specialized laboratory equipment, man- 


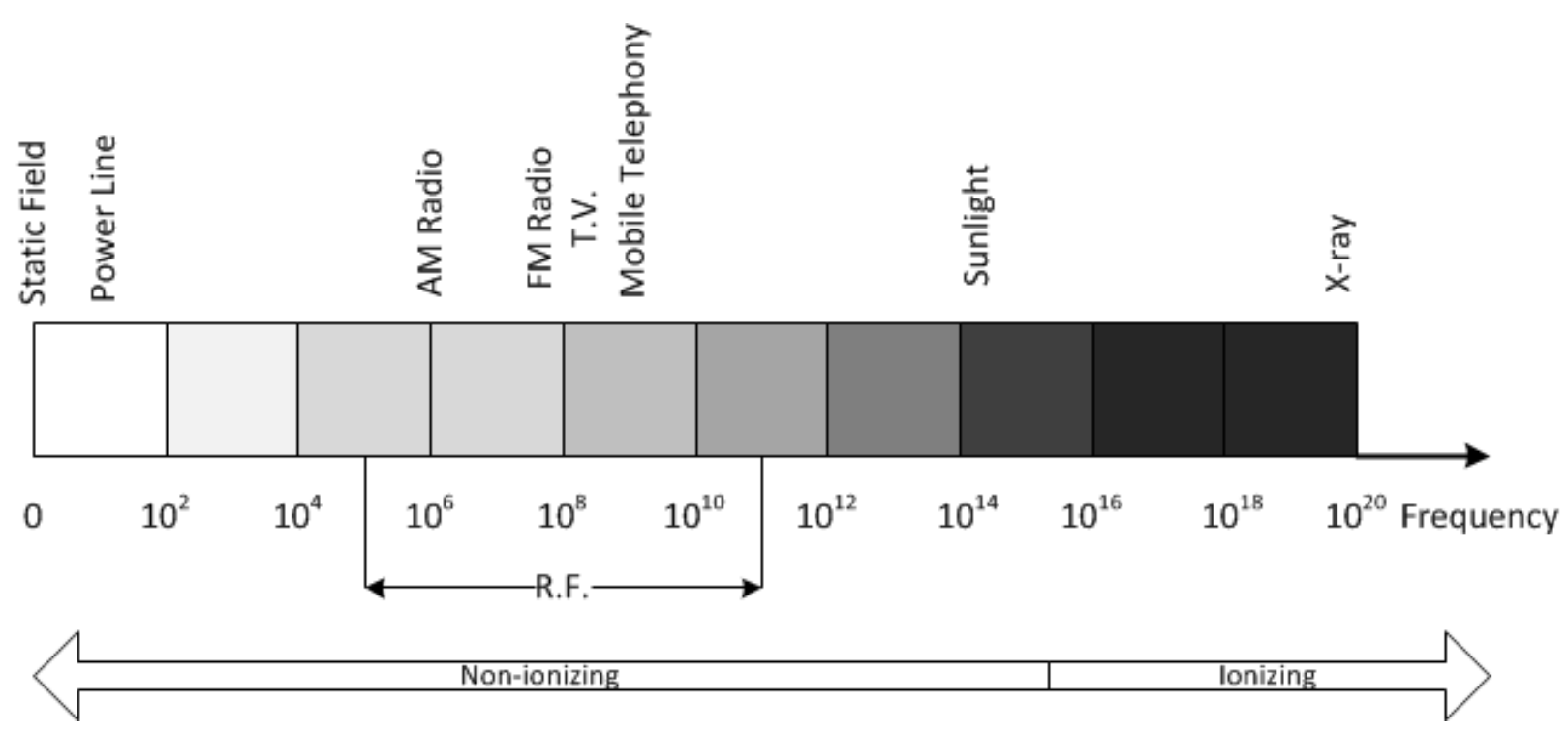

Figure 2. Electromagnetic Spectrum

nequins, electrolytes and the device being measured transmitting at full power while a robot probe scans for SAR measurement. The measured SAR value is then compared with the regulatory prescribed limits.

\section{B. Far-Field and Power Density}

The EM radiation characteristics of an antenna in the farfield are quite different from those in the near-field region. The far-field characterizes radio waves which propagate with the speed of light and for which their electric and magnetic field components are closely associated such that only one of them needs to be evaluated. The far-field region begins at a distance greater than $2 D^{2} / \lambda$ from the transmitting antenna, as it is depicted in Fig. 1, where $D$ is the largest dimension of the antenna (length, width or diameter of the antenna). This distance is referred to as the Fraunhofer distance [19].

$\mathrm{EM}$ radiation in the far-field is commonly measured in terms of power density in $\mathrm{W} / \mathrm{m}^{2}$. Power density is defined as the power per unit area normal to the direction of propagation. Due to the simple relationship between the electric and magnetic fields in this region, the power density can be expressed based on electric field only through the characteristic impedance, such as:

$$
S=E H=\frac{E^{2}}{Z_{0}}=\frac{E^{2}}{377}
$$

where $E, H$ denote the electric and magnetic fields and $Z_{0}$ represents the characteristic impedance [17].

\section{EM RAdiATION IN MOBILE SySTEMS}

\section{A. EM radiation and $R F$ communication spectrum range}

The EM spectrum is a range of EM fields between low frequency and optical EM waves, whereas the RF spectrum covers a frequency range of a few $\mathrm{MHz}$ to some tens of $\mathrm{GHz}$ and is used for broadcasting and telecommunications. FM radio transmitters operate around $100 \mathrm{MHz}$ while television broadcasts between $470 \mathrm{MHz}$ to about $800 \mathrm{MHz}$. For mobile telephony, the GSM, 3G and $4 \mathrm{G}$ systems operate between $900 \mathrm{MHz}$ to $2600 \mathrm{MHz}$ frequency range. The allocation of different frequencies is strictly governed by the international telecommunications union (ITU) and nationally by relevant regulatory agencies. EM radiation is classified into ionizing and non-ionizing radiation [20], as shown in Fig. 2. Ionizing radiation refers to EM radiation with enough energy to remove tightly bound electrons from the orbit of an atom, thus making the atom to become charged or ionized. Non-ionizing radiation does not have enough energy to ionize atoms. However, it has sufficient energy to excite electrons thereby moving them into higher energy state. This paper focuses on non-ionizing radiation as RF waves fall into this category.

\section{B. EM radiation contributing factors in mobile communica- tions}

The mobile communication system consists of a number of BSs each with at least one antenna communicating with several mobile terminals. The geographic area covered by a single BS is known as a cell and it could range from a few meters to several kilometers, depending on the BS antenna used and the traffic density of the area. The mobile communication system is, thus, made of several cells, hence the reason why it is sometimes referred to as a "cellular system". The BS communicates with its serving mobile terminals wirelessly over an RF channel and the operating frequency used by a network depends on the technology deployed and its operational license. A mobile terminal connects to a BS based on the quality of the communication path between them and not necessarily its distance from the BS. Transmission from the mobile terminal to the BS is referred to as the uplink while transmission from the BS to mobile terminals is called the downlink. Each BS in the network is connected to the core network via backhaul links, which could be high-speed fiber cables or microwave links. BSs are typically mounted on towers or on building walls and rooftops. A network provider may deploy additional BSs as the demand for service 
increases, however, the transmit powers of the BSs must be reduced to minimize interference between the cells. The additional deployment increases the network capacity without the need for more radio spectrum.

The mobile communication system has evolved over the years in the form of generations such that each generation has similar operating requirements. The earlier generations of mobile communication systems had very high transmit powers and lower throughput which produced higher levels of EM radiation exposure. However, as the generations evolved, the transmit power requirements became lower, resulting in reduced EM radiation exposure levels [21], and the throughput improved.

According to the generic description of a mobile communication system above, three main factors have been identified regarding the severity of EM exposure: the topology of the networks, e.g. how many BSs the user is surrounded by, the position of the user respective to the BSs and the duration of the exposure.

\section{1) Communication network topology:}

a) Cell deployment: The topology employed by a network provider affects the EM radiation exposure to a large extent. It determines the number, location and transmit power of BSs in the network. A macrocell BS typically has a transmit power of $20 \mathrm{~W}$ per antenna and range of $1 \mathrm{~km}$ to $20 \mathrm{~km}$ [22]. Conventionally, macrocells are used to provide coverage to larger areas. Hence, macrocell BSs are predominantly used to serve areas where the population density is small and have low traffic demand such as suburban and rural areas. In this case, the BS transmits with full power so as to cover as much area as possible. However, this leads to higher levels of EM radiation exposure from the BS. The cells in dense-urban areas are usually smaller (about $100 \mathrm{~m}$ to $500 \mathrm{~m}$ in radius) with lower transmit power of up to $5 \mathrm{~W}$ for accommodating higher demand in capacity due to increased population density [23] and [24]. Even though these BSs have lower transmit power, there is a larger concentration of people close to the BSs and they experience higher levels of EM radiation exposure in the downlink.

b) Distributed antenna topology: Distributed antenna systems (DAS) are multiple geographically separated antennas that are all connected to the same BS within a cell, and are deployed to improve coverage. Distributing spatially separated antennas over an area could reduce large scale shadowing, improve capacity and reduce access distance. The antenna heads in DAS are connected to the BS via dedicated highspeed optical fiber or RF links [25], [26]. It has been shown in [27] and [28] that DAS considerably reduce the transmit power and increase battery life of mobile phones by reducing the access distance, this transmit power reduction has an impact on the levels of EM radiation emission from mobile phones connected to DAS antennas. Given that the transmit power of DAS antenna heads is lower than that of the conventional macrocell BS, this could result in a lower EM radiation exposure from each antenna head when compared to the macrocell BS. However, DAS is likely to increase the downlink EM radiation exposure levels because of the largescale deployment of antenna heads in the network. c) Heterogeneous networks (HetNets): Macrocells are efficient in providing wide coverage for roaming mobile terminals but are less effective in the provision of high data rates per unit area. More signal power is needed to guarantee an acceptable QoS for certain users, especially cell-edge users. The concept of heterogeneous networks (HetNets) has been proposed to tackle this problem. A HetNet consists of small, low power nodes or relay stations overlaid on a macrocell to improve coverage and increase capacity in very dense areas of the network with higher data-rate demands [29]-[33]. The lowpower nodes used in HetNets are mostly picocells, femtocells and relay stations. Picocells are BSs with omnidirectional antennas that have a low transmit power $(250 \mathrm{~mW}$ to $2 \mathrm{~W})$ and are suitable for indoor or outdoor deployment [34]. It has been shown in [35] that joint deployment of macrocells and picocells in urban areas could reduce the total energy consumption of the network by up to $60 \%$. Due to the short distance between the low-power small BSs and the mobile users, there is a considerable potential for reduction in transmit power in both the uplink and downlink directions. Indeed, EM radiation exposure from a mobile phone connected to a small-cell network could be significantly reduced as the mobile phone is closer to the small-cell BS and hence, requires a lower transmit power. Small-cell BSs, like femtocells, have a similar transmit power in comparison with mobile phones [34], which results in considerable reduction of downlink EM radiation exposure. However, the increase in the sources of EM radiation could imply higher EM radiation exposure in the downlink.

2) Location of the user in respect to the BS: EM radiation decreases according to the inverse square law, i.e. $1 /(\text { distance })^{2}$, in the main beam of a BS (far-field). However, the EM radiation is significantly reduced outside the main beam of the BS and the inverse square law does not always hold. At distances very close to a BS antenna on a tower, the EM exposure is very low, but it increases gradually with distance in the direction of the main beam. This is because the energy from BS antennas is directed towards the horizon and is maximum on the ground where the main beam of the BS hits the ground, usually between 50 - $300 \mathrm{~m}$, depending on the BS height, as shown in Fig. 3. The presence of buildings, hills and other obstacles results in greater decrease of EM radiation exposure due to attenuation and reflection. A more realistic decrease in power density by $1 /(\text { distance })^{3.5}$ has been suggested by [36] for BS EM radiation calculations in urban areas. In places with high population density and data traffic, the cell radii of the BSs are usually smaller and EM radiation exposure is also higher due to more BSs, when compared to rural areas. This results in difference in the measurement of EM exposure in different areas [37].

3) Duration of exposure: The EM exposure dose reduces by reducing the EM radiation exposure time or duration of exposure. The authors in [38] concluded that the duration of exposure to EM radiation from a standard GSM mobile phone had an effect on the cognitive functions of humans. It has been shown in [39] that whole-body exposure to a SAR of $4 \mathrm{~W} / \mathrm{kg}$ for 20-30 minutes resulted in a body increase in temperature of up to $0.5^{\circ} \mathrm{C}$. This increase in temperature is, 


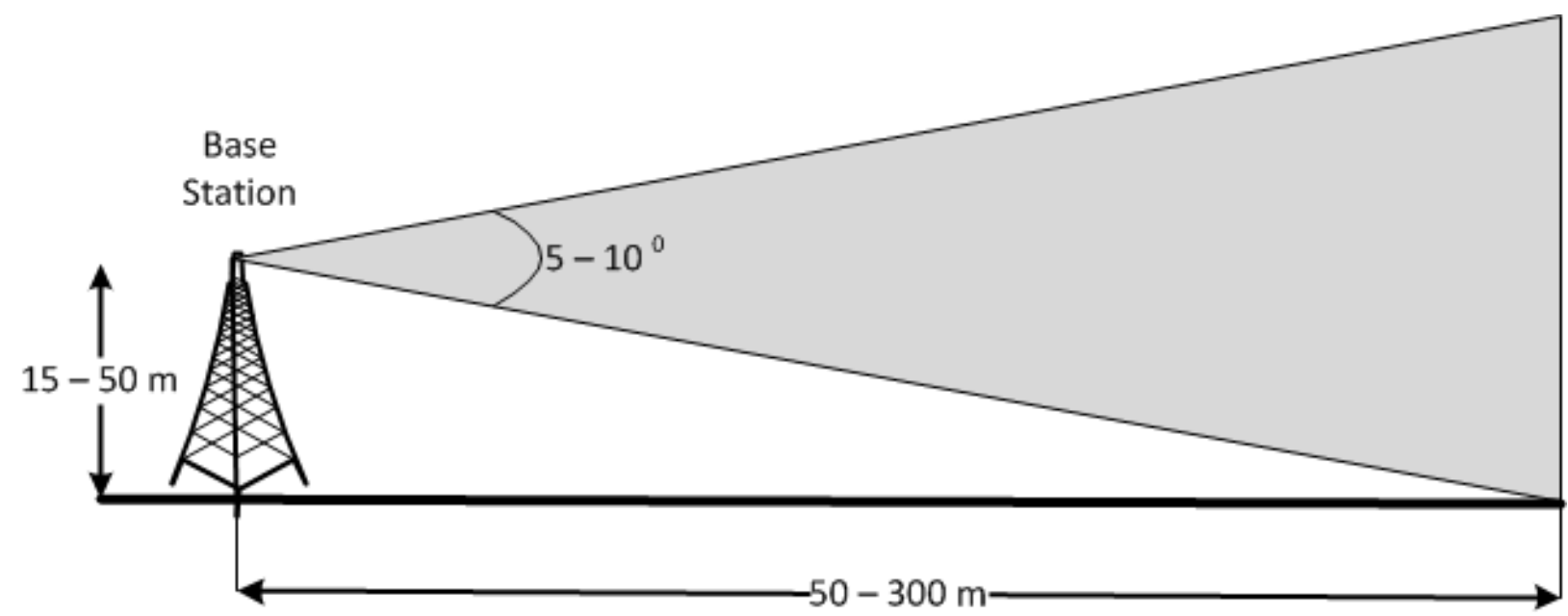

Figure 3. Direction of the main lobe of radiation from a BS antenna.

however, within human thermoregulatory capacity and the EM radiation exposure limit of mobile phones, which is $1.6 \mathrm{~W} / \mathrm{kg}$ in $1 \mathrm{~g}$ of tissue in the United States [40] and $2 \mathrm{~W} / \mathrm{kg}$ in $10 \mathrm{~g}$ of tissue in Europe [17], is below this value.

\section{EM EXPOSURE GUIDELINES AND LIMITS}

This section presents an overview of the dosimetry and guidelines for limiting EM radiation exposure.

\section{A. Dosimetry of EM radiation exposure}

The dosimetry of EM radiation is defined as the evaluation of the energy absorption rate in a body exposed to EM radiation over time, $t$ [41]. The thermal effect is the principal mechanism for assessing the biological and health effects of exposure to EM radiation because increase in body temperature is closely related to the SAR when the exposure period is greater than 6 minutes [17].

The dosimetry of EM radiation exposure can be classified into physical and numerical methods.

- Physical model: This involves the use of a phantom to simulate SAR or current density in the body since it is very difficult to do so in an actual human body using noninvasive methods. Liquid, jelly or gel phantoms are more commonly used to evaluate the EM radiation exposure dosimetry because the materials are easier to prepare and their electrical properties can be easily adjusted to simulate the human body.

- Numerical model: The numerical method involves theoretical calculation of the dosimetry of EM radiation exposure. The finite-difference-time-domain (FDTD) method [42] is currently the most recognized method of SAR calculation [41]. It is based on the discretization of Maxwell's equations. FDTD calculation uses a voxel which is a small volume cube or element of a few millimeters in dimension that corresponds to certain tissues or organs, when similar permittivity and conductivity are assigned.

Studies on human volunteers provide an insight into how EM radiation exposure affects human behavior and health.
The studies are carried out with low EM exposure levels just enough to record minimal changes in the body. Experimental studies carried out on resting humans with a wbaSAR of 1 $\mathrm{W} / \mathrm{kg}$ to $4 \mathrm{~W} / \mathrm{kg}$, which are exposed to EM radiation over a period of 30 minutes, show that such an exposure results in less than $1{ }^{\circ} \mathrm{C}$ rise in body temperature. However, harmful levels of tissue heating have been remarked for SAR values over $4 \mathrm{~W} / \mathrm{Kg}$ [17].

\section{B. EM Exposure Limits}

The International Commission on Non-Ionizing Radiation Protection (ICNIRP) gave guidelines in their reports [17] and [41], on the maximum admissible exposure of people to EM waves of up to $300 \mathrm{GHz}$ for different practical situations and the health consequences of exposure to EM radiation, respectively. These guidelines apply to both occupational and public exposures and are based on laboratory and epidemiological studies. Occupational exposure refers to the EM exposure of adults who are trained and aware of the possible hazards and take necessary precautions. On the other hand, public exposure consists of people of all ages. Typically, members of the public do not take precautions to minimize exposure as they are usually unaware of such exposure. Hence the need for strict restrictions for public exposure compared to occupational exposure, where persons exposed are aware of the potential risks and are trained to take precautions. EM radiation exposure restriction in [17] was specified in terms of SAR (averaged over $t=6$ minutes) and power density (averaged over any $20 \mathrm{~cm}^{2}$ exposed area and $t=68 / f^{1.05}$ minutes), for various frequency ranges. In the $10 \mathrm{MHz}-10$ $\mathrm{GHz}$ frequency range (where mobile communication systems fall), a wbaSAR limit of $0.4 \mathrm{~W} / \mathrm{kg}$ and $0.08 \mathrm{~W} / \mathrm{kg}$, was recommended for occupational and public exposures, respectively, in the report. In terms of power density, limits were set at $50 \mathrm{~W} / \mathrm{m}^{2}$ and $10 \mathrm{~W} / \mathrm{m}^{2}$ for occupational and public exposure, respectively. Some evidence of insignificant changes in brain activity from exposure to radiation from GSM-type mobile phones was reported in [41]. No changes were observed in cognitive function although individuals exposed to EM 
Table I

ICNIRP SAR EXPOSURE LIMITS

\begin{tabular}{|c|c|c|}
\hline & Public & Occupational \\
\hline Whole-body (W/kg) & 0.08 & 0.4 \\
\hline Localized head/trunk (W/kg) & 2 & 10 \\
\hline Localized limbs (W/kg) & 4 & 20 \\
\hline
\end{tabular}

Table II

ICNIRP POWER DENSITY EXPOSURE LIMITS

\begin{tabular}{|c|c|}
\hline & Power Density $\left(\mathrm{W} / \mathrm{m}^{2}\right)$ \\
\hline Public & 10 \\
\hline Occupational & 50 \\
\hline
\end{tabular}

radiation reported subjective symptoms like headaches. The EU has adopted the ICNIRP EM exposure guidelines and all member states have agreed to it in principle. Tables I and II summarize the ICNIRP SAR and power density exposure limits, respectively.

The National Radiological Protection Board (NRPB) of the UK has given guidelines on the limits of exposure to EM from mobile phones, BSs and other sources of EM radiation exposure in its various reports [43]-[45]. The reports were based on extensive review of scientific studies carried out on the effects of exposure to EM radiation. These guidelines have since been adopted and implemented by the UK government. The NRPB reports were based on the possibility of illness or injury due to exposure to EM radiation through the heating of body tissues. Physical quantities like the SAR, electric field strength, magnetic field strength and power density were used to specify EM radiation exposure. The NRPB report [44] suggests a SAR limit of $0.4 \mathrm{~W} / \mathrm{kg}$ for whole body exposure over a period of 15 minutes for occupational exposure and 0.1 $\mathrm{W} / \mathrm{kg}$ for general public exposure, in the frequency range of $10 \mathrm{MHz}-10 \mathrm{GHz}$. Power density limits of 26 to $33 \mathrm{~W} / \mathrm{m}^{2}$ and $100 \mathrm{~W} / \mathrm{m}^{2}$ are set for the $800-900 \mathrm{MHz}$ and $1550-3000 \mathrm{MHz}$ frequency ranges, respectively [44]. Based on sound scientific studies, the NRPB is of the opinion that mobile phone users do not have an increased risk of cancer due to EM exposure [45]. However, these studies remain inconclusive as they do not explore the long-term effects of such exposure. Table III gives the NRPB SAR exposure limits.

A short term mission within the "COST 244bis" action group compiled and explored a large-scale data of EM radiation measurements for different types of GSM BSs and measurement methods in the EU [37]. Key findings, based on data analyzed, are the possibility that the type of BS antennas used, input power of the antennas, distance and shadowing could affect the EM exposure levels at the vicinity of the BS. Also, the EM radiation exposure levels tend to be lower in rural areas than urban areas and the measured EM radiation exposure levels in places accessible were all below the limits set by the ICNIRP.

The European health risk assessment network on EM fields
Table III

NRPB SAR EXPOSURE LIMITS

\begin{tabular}{|c|c|c|}
\hline & Public & Occupational \\
\hline Whole-body (W/kg) & 0.1 & 0.4 \\
\hline Localized (W/kg) & 2 & 10 \\
\hline
\end{tabular}

exposure in its report [46] and more recently the authors in [12], after some extensive surveys of scientific studies on EM measurements and exposure in Europe, observed that there was no noticeable difference in EM exposure levels between countries in the EU, although there is a difference in exposure between rural and urban areas. They also observed that indoor EM radiation exposure is increasing at a much faster rate than outdoor exposure due to the increased use of indoor wireless devices. Perhaps, their most significant finding is that the major sources of EM radiation exposure to the public is from mobile and portable wireless devices and not from fixed transmitters like BSs.

\section{Precautionary Principle}

As a result of the uncertainties of the potential risks of exposure to EM radiation, the WHO has encouraged the adoption of the precautionary principle as a proactive approach to minimize the health effects of exposure to EM radiation [47]. The precautionary principle calls for the adoption of simple, low-cost measures to minimize EM radiation exposure even in the absence of proven risks. These measures include the use of hands-free or earpiece when using mobile phones, minimizing the use of mobile phones, limiting the usage of mobile phones by children, adoption of alternatives to wireless technology for voice and data transmissions and embracing mobile communication systems with low levels of EM radiation exposure, among others [48]-[50].

\section{EM Radiation Exclusion Zones}

As a precautionary approach, EM radiation exclusion zones have been introduced close to BSs with the aim of reducing exposure. The exclusion zone boundary is calculated based on the measured EM radiation from the BS transmitter; indeed, in order to reduce biological effects of EM radiation exposure, distances at which EM radiation are above the exposure limits are confined within the exclusion zone [51]. Distances outside the EM radiation exclusion zone are expected to be below the EM radiation limits and therefore pose no health risks to humans and animals. The Agence Nationale des Fréquences (ANFR), based on ICNIRP recommendations, has proposed safety distances for EM exposure to persons from radio systems (including GSM and UMTS systems) for different configurations of antennas and BS installation [52]. Due to concerns about children being more vulnerable to adverse effects from EM radiation exposure, the Independent Expert Group on Mobile Phones (IEGMP) based on NRPB findings, has suggested that in cases where a BS has to be sited near or within a school, the maximum RF intensity from the BS should 
not be allowed to fall anywhere within the school grounds, as a precautionary approach [53].

\section{EM RAdiation Reduction Techniques}

The four consecutive generations of mobile communication systems have been designed for increasing the spectral efficiency as well as peak data rate. In the fifth generation, it is foreseen that other criteria such as latency, energy efficiency or EM radiation exposure will also have an important role in the design of such communication systems [54]. The next generation will also bring some new concepts to mobile communications, such as heterogenous layout or coordinated multipoint (CoMP) communication, which will have an impact (good or bad) on the EM radiation exposure. With the growing usage of mobile communication devices by the public over longer period of times, the concerns about adverse health effects of EM radiation exposure, from mobile communication systems in particular are rapidly increasing, which brings about the need to investigate techniques for reducing EM radiation from such systems. Moreover, to the best of our knowledge, very few research works have explicitly focused on reducing the EM emission levels of wireless communication systems. The authors in [55] have shown that using lower frequency bands in universal mobile telecommunication systems (UMTS) can reduce the EM radiation density of a BS by about $13 \mathrm{~dB}$ and the transmit power of the primary common pilot channel (CPICH) by about $16 \mathrm{~dB}$.

As there is a direct relationship between transmit power, SAR and EM radiation exposure, we describe in this section some wireless communication techniques that have been proven to reduce the SAR and/or transmit power of mobile communication systems, and hence, EM radiation exposure levels.

\section{A. SAR Shielding}

This technique involves the use of a ferrite material or metamaterial attachment between the mobile phone and the head to reduce SAR. Ferrite materials have very low conductivity which leads to smaller induced currents when exposed to EM waves [56] and they cancel the magnetic field part of an incident EM wave [57]. A metamaterial, on the other hand, is an artificial structure that has negative permittivity and/or negative permeability. A metamaterial is based on the special resonant characteristics of a single cell which produces a negative permittivity and/or negative permeability [58].

A SAR reduction of $47.68 \%$ over 1 gram of tissue was reported in [59], when ferrite materials are used between the mobile phone and human head model. It has been shown in [60] that the use of metamaterials reduce SAR by at least $27 \%$ and $52 \%$, at 900 and $1800 \mathrm{MHz}$, respectively, over 1 gram of tissue, with the antenna performance being less affected.

\section{B. Power Control}

Power control simply refers to adjusting the output transmit power levels of BSs (downlink) or mobile stations (uplink) to maximize the received power of desired signals, improve

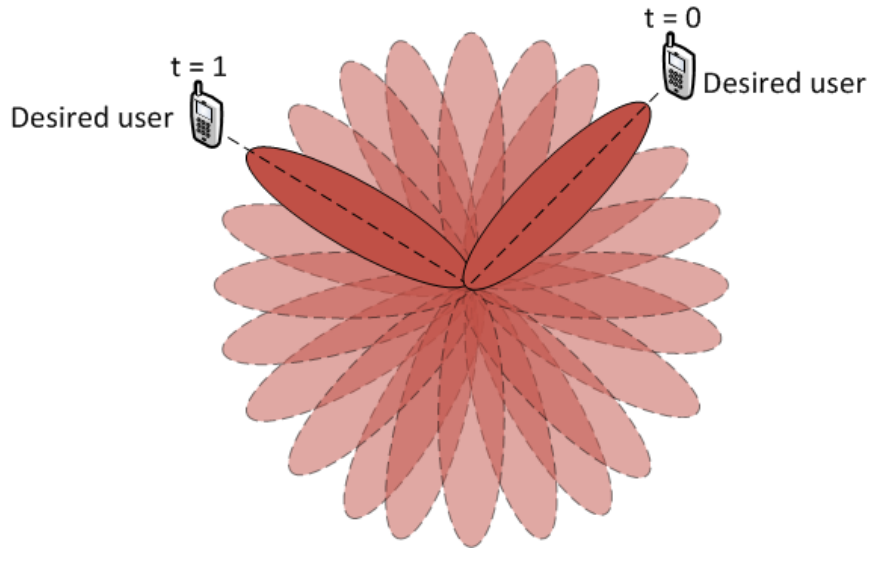

Figure 4. Switched beam pattern.

system capacity as well as coverage, and reduce power consumption as well as interference. Power control is usually utilized in the uplink for minimizing transmit power whilst maintaining the signal to interference ratio (SIR) of each user above a certain threshold [61], [62]. It takes into account shadowing, fading and the distance dependent pathloss between the mobile station and the BS [63]. The mobile station transmits with variable power, based on the channel condition subject to a minimum received power threshold and the maximum transmit power of the mobile station [64]-[66].

Even though power control aims at reducing transmit power levels and reducing interference, it can also reduce, in the process, EM exposure level. However, traditional power control algorithm does not take into account the EM exposure level as an input for optimization. Integrating this criteria into the optimization process has not yet been attempted, even though it is very likely to further reduce the EM exposure, especially in the uplink direction.

\section{Beamforming}

Traditionally, omnidirectional antennas have been used at BSs in cellular communications [67]; because the antenna has no knowledge of the mobile user's location and the state of the environment, it transmits with maximum power in order to reach the desired user and overcome environmental challenges. This, however, leads to excessive power wastage and serves as the main source of co-channel interference. The omnidirectional antenna evolved into sectorized antennas whereby a traditional cell is subdivided into different sectors, each with a directional antenna at the same BS location [68]. Each sector is treated as a single cell and has a considerably greater coverage since the transmit power is focused over a small area. While sectorization reduces co-channel interference across the original cell and improves coverage, it does not address the problem of other-cell interference coming from neighboring cells and unwanted radiation in the network. Adaptive/smart antenna technique is currently the most advanced antenna technology and it employs an array of antennas that form a beam for transmission/reception towards each desired mobile user in the system [67]-[69]. The process of using an array of antennas to focus radiation in a particular direction is referred 
to as beamforming. The main idea of beamforming is that each mobile user's signal is multiplied by complex weights that adjust the magnitude and phase of the signal towards the desired destination. Beamforming has the ability to track the user's exact location using digital signal processing (DSP) algorithms and maximize the received signal at the desired destination while reducing the transmit power, interference and EM radiation exposure [70]-[72]. Thus, beamforming can be considered both as a SAR reduction technique as well as power reduction technique.

Beamforming techniques are classified as switched and adaptive array beamforming based on weight calculation and selection [69], [73].

1) Switched beamforming: The complex weights in switched beamforming are selected from a collection of weights that form beams in predetermined directions. The transmitter switches between the predetermined fixed beams based on received signal strength measurements and selects the one that is best aligned with the desired signal. A typical switched beamforming system consists of multiple beams looking in several directions formed by a phase shifting network as depicted in Fig. 4.

While the switched beam system is easy to implement, it could lead to higher interference when an interfering user is located close to the desired user in the direction of the beam. Moreover, there could be significant performance degradation when the desired user is not at the centre of the beam. Besides, switched beam systems could switch their beams in the direction of multipath signals, away from the desired user as they cannot differentiate multipath interference components from desired signals [68]. This could result in EM radiation exposure to the wrong user(s).

2) Adaptive array beamforming: This is currently the most advanced form of smart antenna technology. Adaptive arrays take advantage of sophisticated signal processing algorithms to effectively locate and track a variety of signals to minimize interference and maximize the signal to interference plus noise ratio (SINR) at the desired destination. The antenna weights are adaptively calculated in real time and the signal in the desired direction maximized by forming a narrow beam in that direction and nulls in undesired directions [69], [67], as shown in Fig. 5. Unlike switched beamforming systems, adaptive array systems can efficiently distinguish between desired, multipath and interfering signals. Hence, antenna weight computation is based on the changes in the location of both the desired and interfering signals [68]. An adaptive antenna technique that is able to adjust its antenna pattern due to changes in channel noise or interference in order to improve the SINR of a desired signal is known as digital beamforming [67].

The authors in [74] and [75] modeled and studied the effect of multi-antenna systems and beamforming on the human head by changing the distance of the antenna elements from the head. In [76], the authors studied the effect of beamforming for SAR mitigation. Their idea was based on putting nulls in the direction of the head thereby reducing the EM radiation absorbed by the head. Results show a peak SAR reduction of at least $10 \mathrm{~dB}$. In [77], the authors proposed a low complexity,

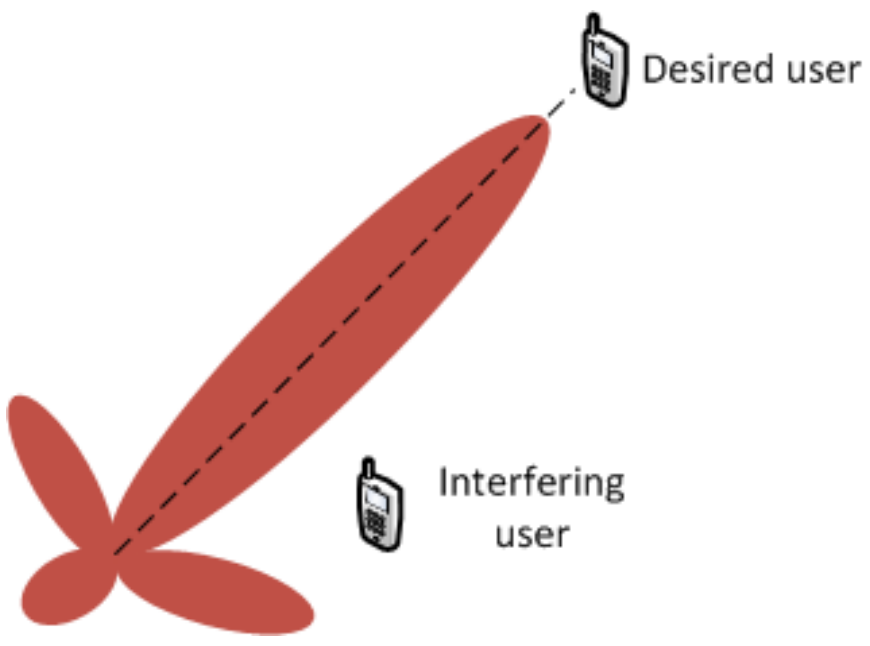

Figure 5. Adaptive antenna system beam formation.

SAR-aware optimal beamformer to minimize EM radiation exposure from mobile terminals. The SAR-aware beamformer was based on their SAR model for multi antenna terminals [78] to maximize SNR with SAR and transmit power constraints. Results show that the optimal beamformer has an SNR gain of up to $4 \mathrm{~dB}$ over the traditional back-off approach for the same SAR value. Similarly, an SNR gain of about 4 $\mathrm{dB}$ was noted over the traditional back-off approach at high transmit power. This means that the algorithm leads to SAR and transmit power reduction by including SAR constraint in the beamforming optimization process.

According to the various works on beamforming previously described, the ability of beamforming to focus signals towards desired users has the advantage of minimizing/eliminating EM radiation towards unintended users. Incorporating EM radiation exposure metrics into the optimization process of joint power allocation and beamforming schemes could significantly reduce EM radiation exposure levels to all users in the system, both in the uplink and downlink directions.

\section{Coordinated Multipoint (CoMP)}

Coordinated multi-point (CoMP) is defined in [79] as the dynamic coordination among multiple geographically separated transmission or reception points. In CoMP, multiple coordinating points cooperate with each other in such a way that transmissions either in the uplink or downlink do not pose serious interference problems or even harness it to improve spectral efficiency and reduce energy consumption of the system [80], [81]. This concept is considered as a means of improving network coverage, spectral efficiency and celledge throughput. It is especially very effective for cell-edge user performance where the cell-edge users could be served by any/all neighboring BSs at the same time, which reduces or even eliminates inter-cell interference [82]-[84]. CoMP usually requires information for scheduling to be available at all BSs participating in CoMP and this could involve very low latency links (in order of milliseconds) to aid the exchange of such information between CoMP nodes. Depending on how the information is shared between coordinating BSs and 


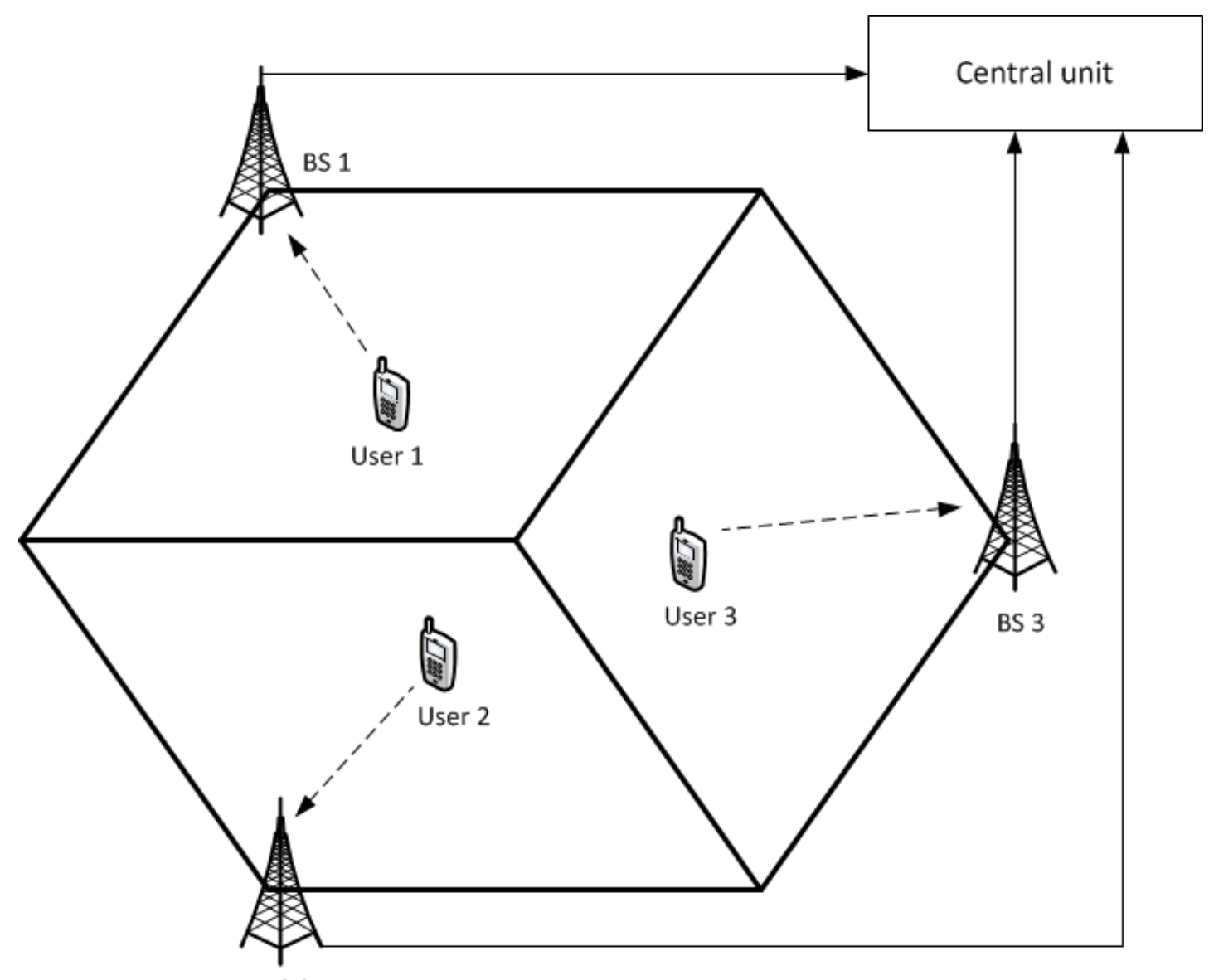

BS 2

Figure 6. Joint processing architecture.

the users, CoMP can be classified into two groups; joint processing and coordinated scheduling/beamforming [85].

1) Joint Processing: In this approach, the channel state information (CSI) of all the users within the CoMP area is stored in a central unit which performs user scheduling and signal processing. Each user measures its CSI with all cooperating BSs and feeds them back to its serving BS which then forwards it to the central unit [82]. Therefore the central unit has the CSI of all the users in the system, which it uses for scheduling and the design of transmission parameters like precoding matrices. The central unit then forwards this information to all coordinating BSs in the system. In joint processing, data intended for a user is jointly transmitted from multiple BSs or from the user to multiple BSs, in the downlink and uplink respectively, to cancel interference and improve the received signal quality. A major drawback of this architecture is the need for high capacity links between each cooperating BS and the central unit [85]. Fig. 6 shows the joint processing architecture.

2) Coordinated Scheduling/Beamforming: In contrast to joint processing, this approach does not have the requirement for a central unit and very low latency links. However, it is based on the assumption that all BSs have identical schedulers and the CSI is available at all cooperating nodes. Each user measures the CSI between it and all the cooperating BSs and feeds back the information to the respective BSs over the wireless channel, as depicted in Fig. 7. Hence, each BS has the CSI between it and all the users in the system and could then perform independent scheduling. This eliminates the infrastructure costs and signaling complexity due to the central unit and the low latency links, respectively [85]. A major drawback of this approach is the handling of errors on the different feedback links. Unlike the joint processing approach where there is only one link for feedback transmission, the number of feedback links in the decentralized approach is equal to the number of cooperating BSs and each of these links is expected to have a different error pattern [82]. The main benefit of the coordinated scheduling/beamforming technique is the reduced need for very high latency coordination links because only information for scheduling/beamforming is needed to be coordinated among the coordinating BSs [79], [82].

Cell-edge communication usually involves the use of higher transmit powers due to the distance from the BS and interference coming from neighboring BSs, bringing about higher EM radiation exposure levels. By using CoMP, lower transmit power could be used due to simultaneous communication with neighboring BSs as well as interference avoidance/reduction capability of CoMP and, in turn, EM radiation exposure could be reduced. Integrating an EM radiation exposure criterion into 


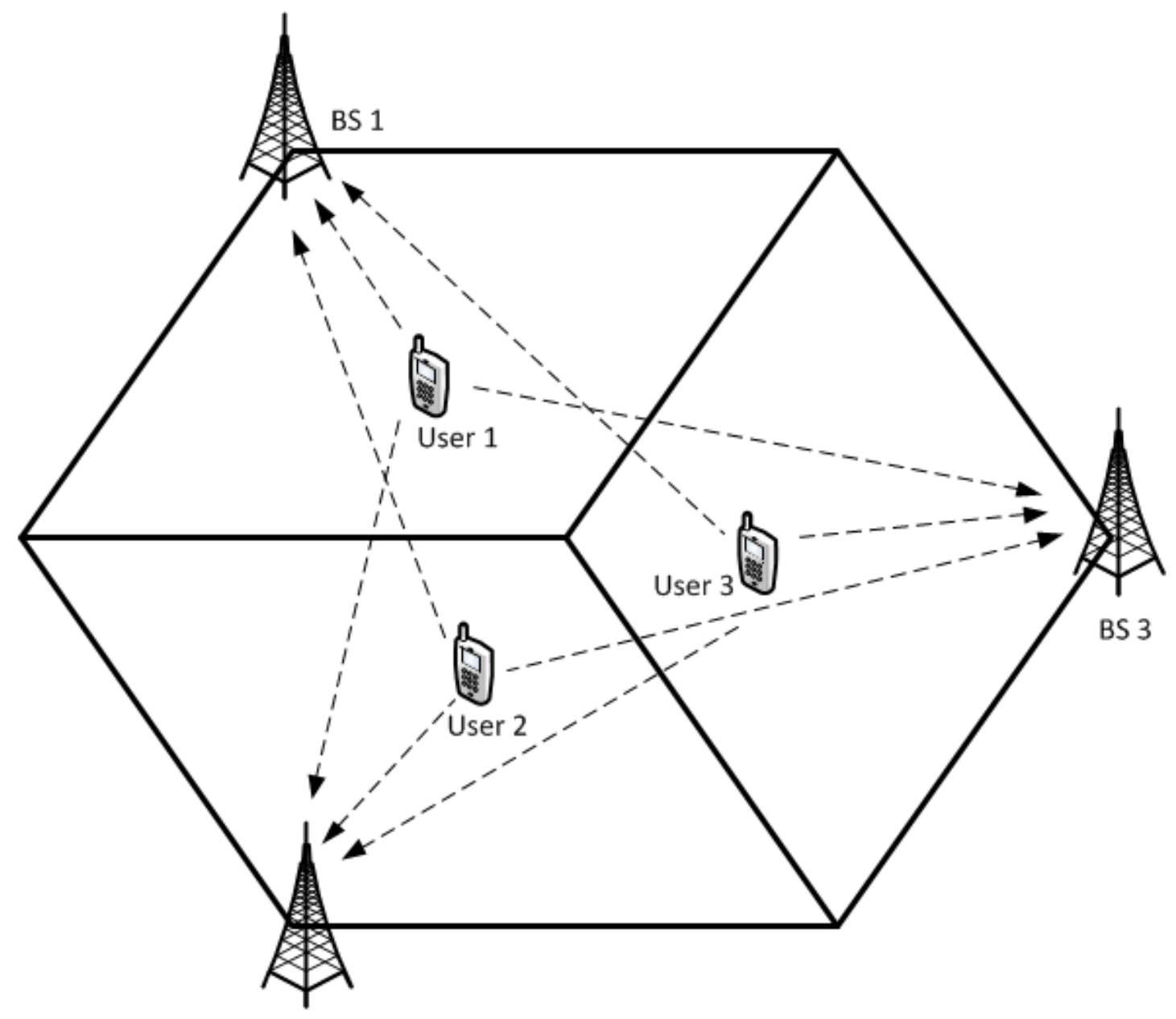

BS 2

Figure 7. Coordinated scheduling/beamforming architecture.

joint power allocation and coordinated beamforming optimization process would bring about a minimization in the EM radiation exposure levels. Similarly, the central unit in joint processing can advice coordinating BSs on their transmission regimes towards desired users, based on the respective pathlosses from the BSs, to minimize the EM radiation exposure level in the far-field while maintaining set SINR thresholds. Beamforming could be used, in the uplink of CoMP, to minimize EM radiation exposure towards the mobile user's head by choosing to communicate with BSs that are in the opposite direction of the mobile user's head. This technique could significantly reduce EM radiation exposure of the user by focusing the signal power away from the user.

\section{E. Massive MIMO}

Massive MIMO, also known as very large MIMO, is an emerging technology whereby a BS with a large number of small antennas, an order of magnitude higher than in current MIMO systems, simultaneously serves a much smaller number of mobile users in the same time-frequency resource. Each antenna in the system uses extremely low power such that the power per antenna is inversely proportional to the number of antennas in the BS [86]. The large number of antennas significantly reduces the transmit power of the BS which could result in lower EM radiation exposure levels [87]-[90]. Fig. 8 depicts the concept of massive MIMO.

Massive MIMO utilizes spatial multiplexing which relies on accurate knowledge of the CSI both in the uplink and downlink. In the uplink, mobile users send pilot signals to the BS which then estimates the CSI between itself and the user terminal. However, obtaining the CSI is a bit more challenging in the downlink as each mobile user would have to estimate the channel responses between itself and each antenna at the BS and feed them back to the BS. This could be computationally and time demanding as the number of antennas at the BS increases. A solution to this problem is to operate massive MIMO systems in the time division duplex (TDD) mode. It relies on the reciprocity between the uplink and downlink channels and, thus, the BS can estimate the CSI by using the uplink pilots [87].

Massive MIMO also has the potential of considerably increasing spectral efficiency through aggressive spatial multiplexing and significantly improving energy-efficiency by using very low-power antennas and amplifiers [86]. It has been shown in [91] that massive MIMO could increase energyefficiency by 3 orders of magnitude by using simple linear 


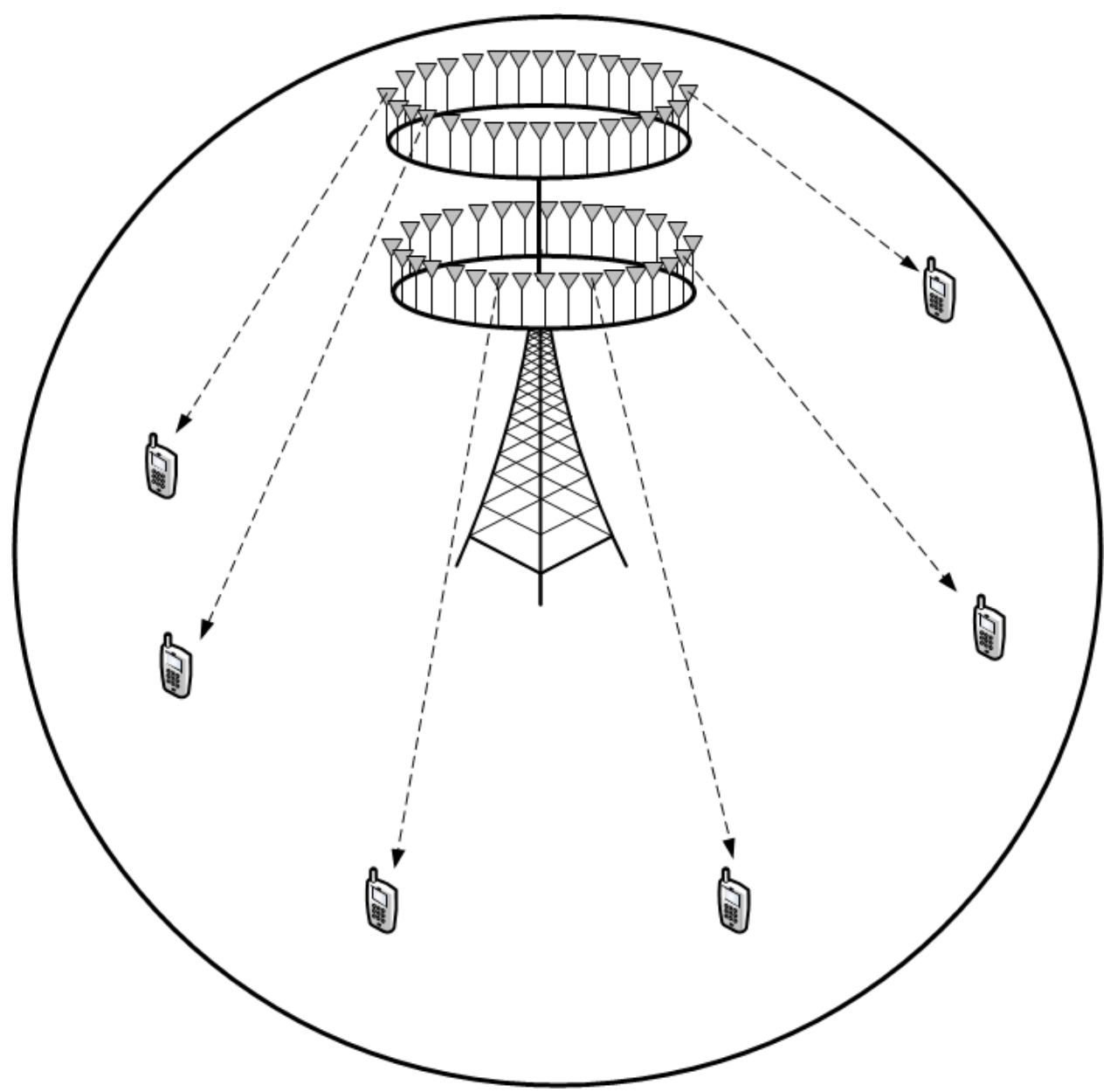

Figure 8. Illustration of a massive MIMO cell with a large number of antennas at the BS.

processing such as maximum ratio combining (MRC) and zero forcing (ZF) at the BS. The authors in [92] have shown that the transmit power of mobile terminals in massive MIMO systems is inversely proportional to the number of antennas at the BS, when there is perfect knowledge of the CSI, and inversely proportional to the square root of the number of antennas at the BS if the information about the channel is imperfect.

Given that massive MIMO is a relatively new technology, there is no research work on massive MIMO that explicitly focuses on reducing EM radiation exposure, to the best of our knowledge. However, the direct relationship between transmit power and EM radiation, and the fact that massive MIMO has the ability to reduce the transmit power of mobile terminals by at least the square-root of the number of antennas at the BS, depending on the CSI quality, imply that implementing massive MIMO could reduce the SAR of mobile terminals by a factor of at least the square-root of the number of antennas at the BS.

\section{CONCLUSION}

A comprehensive survey and tutorial of EM radiation exposure from mobile communication systems has been performed. We presented the basis for limiting EM radiation exposure from mobile systems and an overview of some international projects dedicated to the study and reduction of EM radiation exposure or reduction of transmit powers of mobile communication systems. In order to ease understanding, we analyzed the dosimetry and metrics of EM radiation exposure and subsequently listed adopted international and national guidelines and limits for EM radiation exposure, based on these metrics. Furthermore, we discussed possible approaches of reducing EM radiation exposure from mobile systems by using techniques known to reduce the transmit power and/or SAR of mobile systems.

Based on our analysis of the current literature, we have reached the following conclusions:

1) There are serious concerns about harmful effects of exposure to EM radiation exposure. Although the research works on the adverse health effects of EM radiation exposure from mobile systems are inconclusive, there is a consensus that it has a small possibility of it being carcinogenic. More studies are needed to fully assess the long-term effects of mobile phone use.

2) Accordingly, limits on EM radiation exposure have been set by regulators throughout the world and wireless network operators, as well as mobile terminal manufacturers, have to ensure that they comply with them by continuously carrying out measurements.

3) The amount of EM radiation exposure to a mobile 
communication user depends on the technology of the mobile device, the distance between the mobile device's antenna and the user, the duration of use, and the mobile users distance from the BS.

4) Precautionary approaches like the use of hands-free or earpiece, minimizing the use of mobile phones, limiting the usage of mobile phones by children, etc., could be used to minimize the effect of exposure to EM radiation.

5) Despite these concerns, very limited work has been done on explicit ways of reducing EM radiation exposure from mobile systems as network operators and mobile terminal manufacturers have so far only focused on complying with regulatory limits.

With the advent of the 5th generation of mobile communication systems, a great opportunity presents itself to the wireless communication community to address these concerns. In this regard, we have identified five promising techniques for reducing EM radiation exposure:

a) SAR shielding is a proven technique of reducing the SAR of mobile phones with little degradation of the antenna performance. It has the advantage of being transparent to the network operator, thus, eliminating the need for network upgrades.

b) Power control has the gains of reducing the transmit power and interference in the network, resulting in battery power savings and reduced EM radiation exposure levels.

c) Beamforming has the benefit of reducing interference in the network and EM radiation towards unintended directions by focusing antenna beams in the desired direction. It also leads to improved SINR and a reduction in transmit powers because most of the signal energy is focused in a certain direction, which could lead to lower levels of EM radiation exposure.

d) CoMP achieves improved network coverage, cell-edge throughput, spectral efficiency, energy efficiency and lower transmit power by coordinating multiple geographically separated transmission or reception points. This technique could bring about reduced EM radiation exposure due to the lower transmit powers involved, as well as the possibility of incorporating it with power control and beamforming.

e) Massive MIMO is a promising concept whereby a large number of antennas are located at the BS to serve a much smaller number of mobile users. This leads to improved spectral efficiency, energy efficiency and lower transmit power, which could result in lower EM radiation exposure levels in the network.

\section{ACKNOWLEDGMENT}

This paper reports work (partially) undertaken in the context of the project LEXNET. LEXNET is a project supported by the European Commission in the 7th Framework Programme $\left(\mathrm{GA} \mathrm{n}^{\circ} 318273\right)$. For further information, please visit www. lexnet-project.eu.

\section{REFERENCES}

[1] "Traffic and Market Data Report - On the Pulse of the Networked Society," http://hugin.info/1061/R/1561267/483187.pdf, Ericsson, Tech. Rep., Nov. 2011, [Online]. Accessed Aug. 15, 2014.
[2] D. Mohankumar, "Mobile Phones Radiation," http://www. electroschematics.com/5200/mobile-phone-radiation/, 2010, [Online]. Accessed Aug. 15, 2014.

[3] N. Dutta, "Can Electromagnetic Radiation from Mobile Towers Harm You?" http://www.thehealthsite.com/diseases-conditions/ can-electromagnetic-radiation-from-mobile-towers-harm-you/, Jan. 2013, [Online]. Accessed Aug. 15, 2014.

[4] D. Reid, "French Mobile Mast Debate Raging," news.bbc.co.uk/1/hi/ programmes/click_online/8167716.stm, Jul. 2009, [Online]. Accessed Aug. 15, 2014.

[5] International Agency for Research on Cancer (IARC), "IARC Classifies Radiofrequency Electromagnetic Fields as Possibly Carcinogenic to Humans," Press Release No. 208, May 2011.

[6] The International EMF Project, http://www.who.int/entity/peh-emf/en/, [Online]. Accessed Aug. 15, 2014.

[7] Low EMF Exposure Future Networks (LEXNET), http://www.lexnet.fr/, [Online]. Accessed Aug. 15, 2014.

[8] Energy Aware Radio and Network Technologies (EARTH), http://www. ict-earth.eu, [Online]. Accessed Aug. 15, 2014.

[9] F. Héliot, M. A. Imran, and R. Tafazolli, "Energy-Efficiency Based Resource Allocation for the Orthogonal Multi-User Channel," in Proc. IEEE Vehicular Technology Conference VTC-Fall, Quebec city, Canada, September 2012.

[10] —, "Energy-Efficiency Based Resource Allocation for the Scalar Broadcast Channel," in Proc. IEEE Wireless Commun. and Networking Conference, WCNC. Paris, France: IEEE, April 2012, pp. 193-197.

[11] GreenTouch, http://www.greentouch.org/, [Online]. Accessed Aug. 15, 2014.

[12] P. Gajsek, P. Ravazzani, J. Wiart, J. Grellier, T. Samaras, and G. Thuróczy, "Electromagnetic Field Exposure Assessment in Europe Radiofrequency Fields (10 MHz-6 GHz)," J. Expo. Sci. Environ. Epidemiol., Aug. 2013.

[13] H. Abdulla and R. Badra, "Head Exposure to Cellular Telephones: A system-level study," in Proc. 2010 IEEE Latin-American Conference on Commun. (LATINCOM), Bogota, Sept. 2010, pp. 1-6.

[14] Q. Balzano and A. Faraone, "Human Exposure to Cellular Base Station Antennas," in Proc. 1999 IEEE International Symposium on Electromagnetic Compatibility, vol. 2, Seattle, WA, Aug. 1999, pp. 924-927.

[15] Health Protection Agency Advisory Group on Non-ionising Radiation (AGNIR), "Health Effects from Radio Frequency Electromagnetic Fields, Report of the independent Advisory Group on Nonionising Radiation," http://www.hpa.org.uk/webc/hpawebfile/hpaweb_c/ 1317133827077, Apr. 2012, [Online]. Accessed Aug. 15, 2014.

[16] "IEEE Standard for Safety Levels With Respect to Human Exposure to Radio Frequency Electromagnetic Fields, $3 \mathrm{kHz}$ to $300 \mathrm{GHz}$," IEEE Std C95.1-2005 (Revision of IEEE Std C95.1-1991), pp. 01-238, 2006.

[17] ICNIRP, "Guidelines for Limiting Exposure to Time-Varying Electric, Magnetic, and Electromagnetic Fields (up to 300Ghz)," Health Physics, vol. 4, no. 74, pp. 494-522, 1998.

[18] D. L. Means and K. W. Chan, "Evaluating Compliance with FCC Guidelines for Human Exposure to Radiofrequency Electromagnetic Fields, Additional Information for Evaluating Compliance of Mobile and Portable Devices with FCC Limits for Human Exposure to Radiofrequency Emissions," Office of Engineering and Technology, Federal Communications Commission, Tech. Rep., Jun. 2001.

[19] T. L. Singal, Wireless Communications. New Delhi: Tata McGraw-Hill Education, 2010.

[20] E. B. Podgoršak, Radiation Physics for Medical Physicists. Germany: Springer Berlin Heidelberg, 2006.

[21] G. Ziegelberger et. al., "ICNIRP Statement on EMF-Emitting New Technologies," Health Physics, vol. 94, no. 4, pp. 376-392, 2008.

[22] S. Katiyar, R. K. Jain, and N. K. Agrawal, "R.F. Pollution Reduction in Cellular Communication," International Journal of Scientific and Engineering Research, vol. 3, no. 3, Mar. 2012.

[23] A. Fehske, F. Richter, and G. Fettweis, "Energy Efficiency Improvements Through Micro Sites in Cellular Mobile Radio Networks," in Proc. GLOBECOM Workshops, 2009 IEEE, Honolulu, HI, 2009, pp. 1-5.

[24] S. Katiyar, R. K. Jain, and N. K. Agrawal, "Green Cellular Network Deployment To Reduce RF Pollution," International Journal of Applied Information Systems, vol. 2, no. 6, pp. 21-26, May 2012.

[25] Y. Liu, J. Liu, W. Guo, H. Chen, D. Zheng, and G. Zhang, "Downlink Performance Analysis of Distributed Antenna Systems," in Proc. International Conference on Wireless Commun. and Signal Processing (WCSP), Nanjing, Nov. 2011, pp. 1-5.

[26] W. Choi and J. Andrews, "Downlink Performance and Capacity of Distributed Antenna Systems in a Multicell Environmentapacity of dis- 
tributed antenna systems in a multicell environment," IEEE Transactions on Wireless Commun., vol. 6, no. 1, pp. 69-73, Jan. 2007.

[27] J. Ni, J. Zhang, D. Chen, X. Bi, and Y. Wang, "Distributed Antenna Systems and Their Applications in 4G Wireless Systems," in Proc. IEEE International Conference on Commun. Workshops (ICC), Kyoto, Jun. 2011, pp. 1-4.

[28] A. Obaid and H. Yanikomeroglu, "Reverse-Link Power Control in CDMA Distributed Antenna Systems," in Proc. IEEE Wireless Communs. and Networking Conference, WCNC, vol. 2, Chicago, IL, Sept. 2000, pp. 608-612.

[29] R. Kim, J. S. Kwak, and K. Etemad, "WiMAX Femtocell: Requirements, Challenges, and Solutions," IEEE Commun. Magazine, vol. 47, no. 9, pp. 84-91, 2009.

[30] P. Xia, V. Chandrasekhar, and J. G. Andrews, "Open vs Closed Access Femtocells in the Uplink," IEEE Transactions on Wireless Commun., vol. 9, no. 12, pp. 3798-3809, Dec. 2010.

[31] V. Chandrasekhar, J. Andrews, and A. Gatherer, "Femtocell Networks: A Survey," IEEE Commun. Magazine, vol. 46, no. 9, pp. 59-67, 2008.

[32] J. G. Andrews, H. Claussen, M. Dohler, S. Rangan, and M. C. Reed, "Femtocells: Past, Present, and Future," IEEE Journal on Selected Areas in Commun., vol. 30, no. 3, pp. 497-508, Apr. 2012.

[33] H. Claussen, L. W. Ho, and L. G. Samuel, "An Overview of The Femtocell Concept," Bell Labs Technical Journal, vol. 13, no. 1, pp. 221-245, 2008.

[34] A. Damnjanovic, J. Montojo, Y. Wei, T. Ji, T. Luo, M. Vajapeyam, T. Yoo, O. Song, and D. Malladi, "A Survey On 3Gpp Heterogeneous Networks," IEEE Wireless Commun. Magazine, vol. 18, no. 3, pp. 10 21, Jun. 2011

[35] H. Claussen, L. T. W. Ho, and F. Pivit, "Effects of Joint Macrocell and Residential Picocell Deployment on the Network Energy Efficiency," in Proc. IEEE International Symposium on Personal, Indoor and Mobile Commun., PIMRC, Cannes, Sept. 2008, pp. 1-6.

[36] ETSI, "Radio Network Planning Aspects. ETR 364 (GSM 03.30 version 5.0.0)," European Technical Standards Institute, Tech. Rep., 1996.

[37] U. Bergqvist, "Mobile Telecommunication Base Stations - Exposure to Electromagnetic fields, Report of a Short Term Mission within COST244bis," COST-244bis Short Term Mission on Base Station Exposure, Tech. Rep., 2000.

[38] R. Luria, I. Eliyahu, R. Hareuveny, M. Margaliot, and N. Meiran, "Cognitive Effects of Radiation Emitted by Cellular Phones: The Influence of Exposure Side and Time," Bioelectromagnetics, vol. 30, no. 3, pp. 198-204, Apr. 2009.

[39] F. G. Shellock, D. Schaefer, and J. Crues, "Alterations in Body and Skin Temperatures Caused by Magnetic Resonance Imaging: Is the Recommended Exposure for Radiofrequency Radiation Too Conservative?" British Journal of Radiology, vol. 62, no. 742, pp. 904-909, 1989.

[40] ANSI/IEEE, "IEEE Standard for Safety Levels with Respect to Human Exposure to Radio Frequency Fields, $3 \mathrm{kHz}$ to $300 \mathrm{GHz}$," The Institute of Electrical and Electronics Engineers, Tech. Rep., 1992.

[41] ICNIRP, "Exposure to High Frequency Electromagnetic Fields, Biological Effects and Health Consequences $(100 \mathrm{kHz}-300 \mathrm{GHz})$, International Commission on Non-Ionizing Radiation Protection, Tech. Rep. 16, 2009.

[42] A. Taflove and S. C. Hagness, Computational Electrodynamics: the Finite-Difference Time-Domain Method, 3rd ed. Norwood, MA.: Artech House, 2005.

[43] NRPB, "Statement by NRPB: Restrictions on Human Exposure to Static and Time Varying Electromagnetic Rields and Radiation," National Radiological Protection Board, Tech. Rep. 4(5), 1993.

[44] — "Restrictions on Human Exposure to Static and Time Varying Electromagnetic Fields and Radiation: Scientific Basis and Recommendations for the Implementation of the Board's Statement," National Radiological Protection Board, Tech. Rep. 4(5),7, 1993.

[45] — "Electromagnetic Fields and the Risk of Cancer: Supplementary Report by the Advisory Group on Nonionising Radiation," National Radiological Protection Board, Tech. Rep. 5(2), 77, 1994.

[46] G. Thuróczy, P. Gajsek, T. Samaras, and J. Wiart, "D4: Report on the Level of Exposure (Frequency, Patterns and Modulation) in the European Union Part 1: Radiofrequency (RF) Radiation," European Health Risk Assessment Network on Electromagnetic Fields Exposure, Tech. Rep., Aug. 2010.

[47] L. Kheifets, G. L. Hester, and G. L. Banerjee, "The precautionary principle and emf: implementation and evaluation," Journal of Risk Research, vol. 4, no. 2, pp. 113-125, 2001.

[48] Ng Kwan-Hoong, "Radiation, mobile phones, base stations and your health," Malaysian Communications and Multimedia Commission, Feb. 2005.
[49] U.S. Food and Drug Administration, "RadiationKits and Other Accessories," http://www.fda.gov/ Radiation-EmittingProducts/RadiationEmittingProductsandProcedures/ HomeBusinessandEntertainment/CellPhones/ucm116293.htm, Aug. 2012, [Online]. Accessed Aug. 15, 2014.

[50] World Health Organization (WHO), "Electromagnetic fields and public health: Mobile phones," http://www.who.int/mediacentre/factsheets/ fs193/en/, Jun. 2011, Fact sheet No 193, [Online]. Accessed Aug. 15, 2014.

[51] A. A. De Salles and C. R. Fernández, "Exclusion Zones Close to Wireless Communication Transmitters Aiming to Reduce Human Health Risks," Electromagn. Biol. Med., vol. 25, no. 4, pp. 339-347, 2006.

[52] ANFR, "Guide Technique: Modelisation et perimetres de Securite Pour le Public," Agence Nationale des Fréquences, Tech. Rep. V2, February 2008.

[53] W. Stewart, "Mobile Phones and Health," Independent Expert Group on Mobile Phones, Tech. Rep., Apr. 2000.

[54] N. Cardona et. al, "Scientific Challenges Towards 5G Mobile Communications," COST IC1004 White paper, Tech. Rep., Dec. 2013.

[55] F. Derakhshan, E. Jugl, A. Mitschele-Thiel, and M. Schacht, "Reduction of Radio Emission in Low Frequency WCDMA," in Proc. 5th IEE International Conference on $3 G$ Mobile Commun. Technologies, 2004., London, UK, Oct. 2004, pp. 178-182.

[56] L. Ragha and M. Bhatia, "Evaluation of SAR Reduction for Mobile Phones Using RF Shields," International Journal of Computer Applications, vol. 1, no. 13, pp. 80-85, Jan. 2010.

[57] J. Pretorius, "Design and Manufacture of a Ferrimagnetic Wave Absorber for Cellular Phone Radiations," in Proc. 12th International Symposium on Electron Devices for Microwave and Optoelectronic Applications, 2004. EDMO 2004., Berg-en-Dal, South Africa, Nov. 2004, pp. 119-123.

[58] R. Gómez-Villanueva, H. Jardon-Aguilar, and R. Linares y Miranda, "State of the Art Methods for Low SAR Antenna Implementation," in Proc. 2010 Proceedings of the Fourth European Conference on Antennas and Propagation (EuCAP), Barcelona, Spain, Apr. 2010, pp. 1-4.

[59] M. T. Islam, M. R. I. Faruque, and N. Misran, "Reduction of Specific Absorption Rate (SAR) in the Human Head with Ferrite Material and Metamaterial," Progress In Electromagnetics Research C, vol. 9, pp. 47-58, 2009.

[60] J. Hwang and F. Chen, "Reduction of the Peak SAR in the Human Head with Metamaterials," IEEE Transactions on Antennas and Propagation, vol. 54, no. 12, pp. 3763-3770, Dec. 2006.

[61] G. J. Foschini and Z. Miljanic, "A Simple Distributed Autonomous Power Control Algorithm and its Convergence," IEEE Transactions on Vehicular Technology, vol. 42, no. 4, pp. 641-646, Nov. 1993.

[62] R. D. Yates, "A Framework for Uplink Power Control in Cellular Radio Systems," IEEE Journal on Selected Areas in Commun., vol. 13, no. 7, pp. 1341-1347, Sept. 1995.

[63] A. Simonsson and A. Furuskar, "Uplink Power Control in LTE Overview and Performance, Subtitle: Principles and Benefits of Utilizing Rather than Compensating for SINR Variations," in Proc. 68th IEEE Vehicular Technology Conference, 2008. VTC 2008-Fall, Calgary, BC, Sept. 2008, pp. 1-5.

[64] 3GPP, "Evolved Universal Terrestrial Radio Access (E-UTRA); Physical Layer Procedures," 3rd Generation Partnership Project (3GPP), TS 36.213, Sept. 2008

[65] B. Muhammad and A. Mohammed, "Performance Evaluation of Uplink Closed Loop Power Control for LTE System," in Proc. 70th IEEE Vehicular Technology Conference Fall (VTC 2009-Fall), Anchorage, AK, Sept. 2009, pp. 1-5.

[66] J. Whitehead, "Signal-Level-Based Dynamic Power Control for CoChannel Interference Management," in Proc. 43rd IEEE Vehicular Technology Conference, New Jersey, USA, May 1993, pp. 499 - 502.

[67] I. Stevanovic, A. Skrivervik, and J. R. Mosig, "Smart Antenna Systems for Mobile Communications," Ecole Polytechnique Federale De Lausanne, Tech. Rep., Jan. 2003.

[68] R. K. Jain, S. Katiyar, and N. K. Agrawal, "Smart Antenna for Cellular Mobile Communication," VSRD International Journal of Electrical, Electronics and Commun. Eng., vol. 1, no. 9, pp. 530-541, Nov. 2011.

[69] D. Nowicki and J. Roumeliotos, Smart Antenna Strategies. The Mobile Communications International, April 1995.

[70] A. Dammann, R. Raulefs, and S. Kaiser, "Beamforming in Combination with Space-Time Diversity for Broadband OFDM Systems," in Proc. IEEE International Conference on Commun., ICC, New-York, USA, Apr. 2002, pp. 165-171. 
[71] M. Olfat, F. Rashid-Farrokhi, and K. Liu, "Power Allocation for OFDM using Adaptive Beamforming over Wireless Network," IEEE Transactions on Commun., vol. 53, no. 3, pp. 505-514, Mar. 2005.

[72] B. Y. Song, R. L. Cruz, and B. Rao, "Simple Joint Beamforming, and Power Control Algorithm for Multi-User MIMO Wireless Networks," in Proc. 2004 IEEE Vehicular Technology Conference - VTC 2004 Fall, Los Angeles, CA, Sept. 2004, pp. 247-251.

[73] M. Kiruba, "Smart Antennas for Wireless Mobile Communication," ITtoolbox Wireless- 15592, Tech. Rep., 22 Oct. 2004.

[74] C. Qiang, Y. Komukai, and K. Sawaya, "SAR investigation of array antennas for mobile handsets," IEICE Transactions on Communications, vol. 90, no. 6, pp. 1354-1356, 2007.

[75] K. R. Mahmoud, M. El-Adawy, S. M. Ibrahem, R. Bansal, and S. H. Zainud-Deen, "Investigating the Interaction Between A Human Head and A Smart Handset for 4G Mobile Communication Systems," Progress In Electromagnetics Research C, vol. 2, pp. 169-188, 2008.

[76] M. Mangoud, R. Abd-Alhameed, N. McEwan, P. Excell, and E. Abdulmula, "SAR Reduction for Handset With Two-Element Phased Array Antenna Computed Using Hybrid MOM/FDTD Technique," Electronics Letters, vol. 35, no. 20, pp. 1693-1694, 1999.

[77] D. Ying, D. J. Love, and B. M. Hochwald, "Beamformer Optimization With a Constraint on User Electromagnetic Radiation Exposure," in Proc. 47th IEEE Annual Conference on Information Sciences and Systems (CISS), Baltimore, MD, Mar. 2013, pp. 1-6.

[78] B. M. Hochwald and D. J. Love, "Minimizing Exposure to Electromagnetic Radiation in Portable Devices," in Proc. IEEE Information Theory and Applications Workshop (ITA), 2012, San Diego, CA, Feb. 2012, pp. 255-261.

[79] 3GPP, "Further Advancements for EUTRA: Physical Layer Aspects," 3rd Generation Partnership Project (3GPP), TR 36.814, Jun. 2009.

[80] F. Héliot, M. A. Imran, and R. Tafazolli, "Energy Efficiency Analysis of Idealized Coordinated Multi-Point Communication System," in Proc. IEEE VTC spring 2011, May 2011, pp. 1-5.

[81] Q. Cui, B. Luo, and X. Huang, "Joint Power Allocation Solutions for Power Consumption Minimization in Coordinated Transmission System," in Proc. 2011 IEEE Globecom Workshops, Houston, TX, Dec. 2011, pp. 452-457.

[82] P. Chand, R. Mahapatra, and R. Prakash, "Energy Efficient Coordinated Multipoint Transmission and Reception Techniques - A Survey," International Journal of Computer Networks and Wireless Commun. (IJCNWC), vol. 3, no. 4, Aug. 2013.

[83] C. Kirallah, D.Vakobratovic, and J.Thompson, "On Energy Efficiency of Joint Transmission Coordinated Multi-Point in LTE-Advanced," in Proc. International ITG Workshop on Smart Antenna (WSA), 2012.

[84] O. Onireti, F. Heliot, and M. Imran, "On the Energy EfficiencySpectral Efficiency Trade-Off in the Uplink of CoMP System," IEEE Transactions on Wireless Commun., vol. 11, no. 2, pp. 556-561, Feb. 2012.

[85] M. Bengtsson et. al., "D1.4 Intial Report on Advanced Multiple Antenna Systems," Wireless World Initiative New Radio WINNER Project, TR CELTIC/CP5-026 WINNER+, Jan. 2009.

[86] F. Rusek, D. Persson, B. K. Lau, E. G. Larsson, T. L. Marzetta, O. Edfors, and F. Tufvesson, "Scaling up MIMO: Opportunities and Challenges with Very Large Arrays," IEEE Signal Processing Magazine, vol. 30, no. 1, pp. 40-60, 2013.

[87] E. G. Larsson, F. Tufvesson, O. Edfors, and T. L. Marzetta, "Massive MIMO for Next Generation Wireless Systems," CoRR, vol. abs/1304.6690, 2013.

[88] A. L. Anderson and M. A. Jensen, "Beamforming in Large-Scale MIMO Multiuser Links Under A Per-Node Power Constraint," in Proc. 2012 International Symposium on Wireless Commun. Systems (ISWCS), Paris, France, Aug. 2012, pp. 821-825.

[89] E. Björnson, M. Kountouris, and M. Debbah, "Massive MIMO and Small Cells: Improving Energy Efficiency by Optimal Soft-Cell Coordination," in Proc. 20th International Conference on Telecommunications (ICT'13), Casablanca, Morocco, May 2013, pp. 1-5.

[90] J. Kang, J. Kang, N. Lee, B. M. Lee, and J. Bang, "Minimizing Transmit Power for Cooperative Multicell System with Massive MIMO," in Proc. 2013 IEEE Consumer Commun. and Networking Conference (CCNC), Las Vegas, NV, Jan. 2013, pp. 438-442.
[91] H. Q. Ngo, E. G. Larsson, and T. L. Marzettat, "Energy and Spectral Efficiency of Very Large Multiuser MIMO Systems," IEEE Transactions on Commun., vol. 61, no. 4, pp. 1436-1449, Apr. 2013.

[92] _ - "Uplink Power Efficiency of Multiuser MIMO with Very Large Antenna Arrays," in Proc. 2011 49th Annual Allerton Conference on Commun., Control, and Computing (Allerton), Monticello, IL, Sept. 2011, pp. 1272-1279.

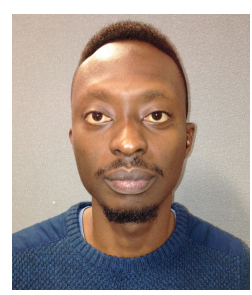

Yusuf Abdulrahman Sambo (S'11) received the B.Eng. degree in Electrical Engineering from Ahmadu Bello University, Zaria, Nigeria, in 2010 and the M.Sc. degree (with distinction) in Mobile and Satellite Communications from the University of Surrey in 2011, where he is currently working towards the Ph.D. degree with the Institute for Communication Systems (ICS). His main research interests include EM radiation exposure reduction, resource allocation, energy efficiency, MIMO and cooperative communication.

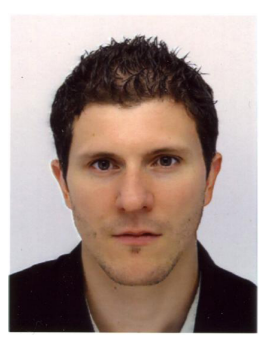

Fabien Héliot (S'05-M'07) received the M.Sc. degree in Telecommunications from the Institut Supérieur de l'Electronique et du Numerique (ISEN), Toulon, France, and the Ph.D. degree in Mobile Telecommunications from King's College London, in 2002 and 2006, respectively. He is currently a Lecturer at the Institute for Communication Systems (ICS) of the University of Surrey, formerly known as CCSR. He has been actively involved in European Commission (EC) funded projects such as FIREWORKS, ROCKET, SMART-Net projects and more recently in the award-winning EARTH project. He is currently involved in the LexNet and 5GIC projects, a EC funded project on reducing wireless communication induced electromagnetic (EM) exposure to human and a UK funded project on shaping the future of wireless communication, respectively. His main research interests are energy efficiency, EM exposure reduction, cooperative communication, MIMO, and radio resource management. He received an Exemplary Reviewer Award from IEEE COMMUNICATIONS LETTERS in 2011.

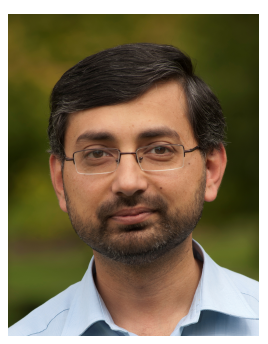

Muhammad Ali Imran (M'03, SM'12) received the M.Sc. (with Distinction) and Ph.D. degrees from Imperial College London, UK, in 2002 and 2007, respectively. He is currently a Reader in the Institute for Communication Systems (ICS), home of 5G Innovation Centre, at the University of Surrey, UK. In this role, he is leading a number of international research projects encompassing the areas of energy efficiency, fundamental performance limits, sensor networks and self-organising cellular networks. He is also leading the new physical layer work area for 5G innovation centre at Surrey. He has a global collaborative research network spanning both academia and key industrial players in the field of wireless communications. He has supervised 18 successful $\mathrm{PhD}$ graduates and published over 150 peer-reviewed research papers including more than 20 IEEE Journals. His research interests include the derivation of information theoretic performance limits, energy efficient design of cellular system and learning/selforganising techniques for optimisation of cellular system operation. He is an editor of IET Communications and a guest editor of special issues in IEEE Communications Magazine and IEEE Wireless Communications Magazine. $\mathrm{He}$ is a senior member of IEEE and a Fellow of Higher Education Academy (FHEA), UK. He has won the Faculty Learning and Teaching Award 2014 and has been awarded the 2014 IEEE Communications Society Fred W. Ellersick Prize. 